\title{
CONSTRUCTION OF A SPECTRALLY STABLE SELF-SIMILAR BLOWUP SOLUTION TO THE SUPERCRITICAL COROTATIONAL HARMONIC MAP HEAT FLOW
}

\author{
PAWEŁ BIERNAT AND ROLAND DONNINGER
}

\begin{abstract}
We prove the existence of a (spectrally) stable self-similar blow-up solution $f_{0}$ to the heat flow for corotational harmonic maps from $\mathbb{R}^{3}$ to the three-sphere. In particular, our result verifies the spectral gap conjecture stated by one of the authors and lays the groundwork for the proof of the nonlinear stability of $f_{0}$. At the heart of our analysis lies a new existence result of a monotone self-similar solution $f_{0}$. Although solutions of this kind have already been constructed before, our approach reveals substantial quantitative properties of $f_{0}$, leading to the stability result. A key ingredient is the use of interval arithmetic: a rigorous computer-assisted method for estimating functions. It is easy to verify our results by robust numerics but the purpose of the present paper is to provide mathematically rigorous proofs.
\end{abstract}

\section{INTRODUCTION}

Let $(M, g)$ and $(N, h)$ be two Riemannian manifolds with metrics $g$ and $h$, respectively. Harmonic maps $F: M \rightarrow N$ are defined as critical points of the functional

$$
S(F)=\int_{M} g^{j k} \partial_{j} F^{a} \partial_{k} F^{b} h_{a b} \circ F,
$$

which is a generalization of the classical Dirichlet energy. Harmonic maps have a number of applications in physics, e.g. in the description of ferromagnetism. Given two manifolds $M$ and $N$, a natural mathematical problem is to construct or, ideally, characterize harmonic maps from $M$ to $N$. A classical device for that purpose is the associated harmonic map heat flow which may be used to deform an arbitrary map to a harmonic one [11]. This works well under certain assumptions on the curvature but in general fails due to the onset of singularities in finite time. The goal is then to develop a sufficiently good understanding of singularity formation in order to continue the flow beyond the singularity in a suitable manner. To this end it is necessary to understand the generic blowup behavior of the flow.

In many cases it is possible to demonstrate finite-time blowup by constructing explicit solutions. However, the relevance of these particular examples with respect to generic behavior is strongly dependent on their stability. The aim of this paper is to study stable singularities of the harmonic map heat flow in the case $M=N=\mathbb{S}^{3}$. As a matter of fact, the curvature of the base manifold $M$ is irrelevant for the asymptotic behavior near a singularity and thus, for simplicity, we may equally well set $M=\mathbb{R}^{3}$. Furthermore, we restrict ourselves to

Roland Donninger is supported by the Alexander von Humboldt Foundation via a Sofja Kovalevskaja Award endowed by the German Federal Ministry of Education and Research. Partial support by the Deutsche Forschungsgemeinschaft (DFG), CRC 1060 'The Mathematics of Emergent Effects', is also gratefully acknowledged.

${ }^{1}$ Einstein's summation convention is in force. 
corotational maps $F: \mathbb{R}^{3} \rightarrow \mathbb{S}^{3}$ which are of the form $F(r, \theta, \varphi)=(u(r), \theta, \varphi)$, where $(r, \theta, \varphi)$ are the standard spherical coordinates on $\mathbb{R}^{3}$, and we use hyperspherical coordinates on $\mathbb{S}^{3}$. The heat flow for such maps is then described by the parabolic Cauchy problem

$$
\left\{\begin{array}{l}
\partial_{t} u(r, t)=\partial_{r}^{2} u(r, t)+\frac{2}{r} \partial_{r} u(r, t)-\frac{\sin (2 u(r, t))}{r^{2}} \\
u(r, 0)=r v_{0}(r), \quad\left\|v_{0}\right\|_{\infty}<\infty
\end{array}\right.
$$

where now $u:[0, \infty) \times[0, \infty) \rightarrow \mathbb{R}$ is time-dependent. An analogous symmetry reduction is possible for maps $F: \mathbb{R}^{d} \rightarrow \mathbb{S}^{d}$.

As in [18, 1, 22, 7, 19], we focus on the blow-up scenario, where a solution $u(r, t)$, starting from smooth initial data, develops a rapidly increasing gradient at $r=0$,

$$
\lim _{t \rightarrow T-} \partial_{r} u(0, t)=\infty .
$$

Here, $T>0$ is the blow-up time. One finds that in dimensions $d=3,4,5,6$ the gradient increases according to the parabolic scaling symmetry of the equation $(r \rightarrow \lambda r$ and $T-t \rightarrow$ $\left.\lambda^{2}(T-t)\right)$, so that $\partial_{r} u(0, t) \propto(T-t)^{-\frac{1}{2}}$. This is referred to as self-similar blow-up and has been studied numerically [3] and rigorously [13, 12]. In higher dimensions $d>7$, the blow-up takes a more complicated form as described in [2, 5, 6].

The harmonic map heat flow bares a striking similarity to other parabolic equations, also displaying a blow-up scenario, such as Yang-Mills flow and semilinear heat equation. In fact, one of the authors and Schörkhuber have recently proved the nonlinear stability of a self-similar solution for the Yang-Mills flow [10]. The proof in [10] relies on a closed-form expression for the self-similar profile to solve the spectral stability problem. Such a closedform expression is unavailable for the harmonic map flow but in this paper we show how to circumvent this issue. Similar approaches were used in [9, 8, which inspired this paper.

As already noted, the Cauchy problem (1.1) has long been known [12, 13] to possess self-similar solutions of the form

$$
u(r, t)=f\left(\frac{r}{\sqrt{T-t}}\right)
$$

where $f$ solves the boundary value problem

$$
f^{\prime \prime}(y)+\left(\frac{2}{y}-\frac{y}{2}\right) f^{\prime}(y)-\frac{1}{y^{2}} \sin (2 f(y))=0, \quad y \geq 0, \quad f(0)=0, \quad f(\infty)=\text { const } .
$$

In [12], it is proved that there exists a countable family of solutions, denoted by $\left\{f_{n}\right\}_{n=0,1, \ldots}$, indexed by their number of intersections with $\pi / 2$. Each solution $f_{n}$ is shown to have $n-1$ extrema and $n$ intersections with $\pi / 2$. In [13], one finds a related existence result: it is proved that there exists a monotone solution to (1.2), which crosses $\pi / 2$ exactly once. In addition to the rigorous results, a family of self-similar solutions, with the same qualitative properties as the ones from [12], was found numerically in [3]. On top of the existing results, our paper adds yet another proof of existence of a monotone self-similar solution.

Theorem 1.1. Let

$$
\widetilde{f}_{0}(y):=2 \arctan \left(\sum_{n=0}^{14}\left(f_{0}\right)_{n} T_{2 n+1}\left(\frac{y}{\sqrt{2+y^{2}}}\right)\right),
$$


with coefficients $\left(f_{0}\right)_{n}$ given in Table B.1 and $T_{n}$ being the standard Chebyshev polynomials. There exists a monotone solution $f_{0} \in C^{\infty}([0, \infty))$ to (1.2) such that

$$
\left\|f_{0}-\widetilde{f}_{0}\right\| \leq 5 \cdot 10^{-4}
$$

where the norm $\|\cdot\|$ is given by

$$
\|f\|:=\left\|p_{1} f\right\|_{L^{\infty}(0, \infty)}+\left\|p_{3} f^{\prime}\right\|_{L^{\infty}(0, \infty)}, \quad p_{1}(y)=\frac{\sqrt{2+y^{2}}}{\sqrt{2} y}, \quad p_{3}(y)=\frac{\left(2+y^{2}\right)^{3 / 2}}{2 \sqrt{2}} .
$$

Remark 1.2. Interestingly, there is still no uniqueness result for self-similar solutions: we do not know if the family of solutions found in [12] is exhaustive. Even worse, strictly speaking we do not know if the solutions $f_{0}$ found in this and other papers [13, 12] are the same (which, however, is very reasonable to assume). Hopefully, a result similar to [17] can be established in the future. From now on, to avoid confusion, whenever we refer to $f_{0}$ we mean the solution from Theorem 1.1.

In addition to the existence result we have the following technical proposition to describe some qualitative properties of $f_{0}$ needed in the proof of nonlinear stability in [4].

Proposition 1.3. Any solution $f \in C^{\infty}([0, \infty))$ to (1.2) has vanishing even derivatives at $y=0$, that is,

$$
f^{(2 k)}(0)=0, \quad k \in \mathbb{N}_{0}
$$

and for each $k \in \mathbb{N}$ there exists a constant $C_{k}>0$ such that

$$
\left|f^{(k)}(y)\right| \leq C_{k} y^{-2-k}
$$

for all $y \geq 1$.

Although Theorem 1.1 seems superfluous at first glance (we already mentioned two other proofs finding similar solutions), our approach represents a significant advantage over the previous ones: the explicit form of $\tilde{f}_{0}$ allows us to rigorously show that our solution is spectrally stable (modulo a gauge mode), see Theorem [1.6 below for the precise meaning of this. Indeed, the linear stability of a monotone solution was conjectured in [3], where it is claimed that a (self-adjoint) linear operator associated to it has no unstable eigenvalues (i.e. eigenvalues in the interval $(-\infty, 0])$, apart from a gauge eigenvalue $\lambda=-1[2$. The latter refers to an eigenvalue that is related to the time translation symmetry of Eq. (1.1) and which does not constitute a "real" instability. The existence of the gauge eigenvalue is easily seen by noting that $y f_{0}^{\prime}(y)$ is a corresponding eigenfunction, see below. The claim of linear stability in [3] was supported by a simple Sturm oscillation argument, which excludes eigenvalues $\lambda \in(-\infty,-1)$, along with a numerical test to exclude $\lambda \in(-1,0]$. The Sturm oscillation argument can be made rigorous very easily (and in fact we use it here), but the rigorous exclusion of eigenvalues in the interval $(-1,0]$ seems impossible without additional quantitative information on the profile of the self-similar solution. Our main motivation for establishing Theorem 1.1 in its particular form is to provide this missing quantitative information.

\footnotetext{
${ }^{2}$ One can find an even stronger conjecture in [3], namely that $f_{0}$ is a generic solution attractor for a large set of data. Our result serves as the first step in proving this conjecture.
} 
To analyze the stability of a self-similar solution $f_{0}$, let us consider equation (1.1) in self-similar variables

$$
\begin{array}{r}
s=-\log (T-t), \quad y=\frac{r}{\sqrt{T-t}}, \quad f(y, s)=u(r, t), \\
\partial_{s} f=\partial_{y}^{2} f+\left(\frac{2}{y}-\frac{y}{2}\right) \partial_{y} f-\frac{1}{y^{2}} \sin (2 f) .
\end{array}
$$

Evidently, $f_{0}$, being a solution to (1.2), automatically leads to a stationary solution $f(y, s)=$ $f_{0}(y)$ to (1.4) and thus a solution global in time $s=-\log (T-t)$. Let us set $f(y, s)=$ $f_{0}(y)+e^{-\lambda s} w(y)$ and linearize in $w$, to get the spectral problem $\left(\lambda-\mathcal{A}_{0}\right) w=0$ for the operator

$$
\begin{aligned}
\mathcal{A}_{0} w(y) & =-\frac{1}{\rho(y)} \partial_{y}\left[\rho(y) \partial_{y} w(y)\right]+\frac{2 \cos \left(2 f_{0}(y)\right)}{y^{2}} w(y) \\
& =-\frac{1}{\rho(y)} \partial_{y}\left[\rho(y) \partial_{y} w(y)\right]+\frac{2}{y^{2}} w(y)+V_{0}(y) w(y) \\
\rho(y) & =y^{2} e^{-\frac{y^{2}}{4}}, \quad V_{0}(y)=\frac{-4 \sin \left(f_{0}(y)\right)^{2}}{y^{2}} .
\end{aligned}
$$

Consider $\mathcal{A}_{0}$ as an operator on the weighted $L^{2}$-space

$$
\mathcal{H}:=L_{\rho}^{2}(0, \infty)
$$

with domain $C_{0}^{\infty}(0, \infty)$. In this setting, $\mathcal{A}_{0}$ is densely defined and symmetric. It is not hard to see that the possible endpoint behavior of solutions to $\mathcal{A}_{0} w=0$ at 0 is $w(y) \propto y$ and $w(y) \propto y^{-2}$. Consequently, only the recessive solution belongs to $\mathcal{H}$ and thus, by the Weyl alternative, $\mathcal{A}_{0}$ is in the limit-point case at 0 . Similarly, close to $\infty$ we have the two behaviors $w(y) \propto 1$ and $w(y) \propto y^{-1} e^{\frac{y^{2}}{4}}$ implying that $\mathcal{A}_{0}$ is limit-point at infinity, too. By Theorem X.7 from [20], we conclude that $\mathcal{A}_{0}$ is essentially self-adjoint (the theorem in [20] applies to operators of the from $-\frac{d^{2}}{d x^{2}}+V(x)$ but it is easy enough to reduce $\mathcal{A}_{0}$ to that form by changing variables to $\left.v(y)=\rho(y)^{\frac{1}{2}} w(y)\right)$. Furthermore, from the endpoint behavior it follows that the closure of $\mathcal{A}_{0}$ has compact resolvent. In summary, we arrive at the following basic result on the spectral theory of $\mathcal{A}_{0}$.

Proposition 1.4. The operator $\mathcal{A}_{0}$ is essentially self-adjoint and the spectrum of its closure consists of a countable number of real, simple eigenvalues.

Definition 1.5. From now on we will denote by $\mathcal{A}_{0}$ the unique self-adjoint extension on $L_{\rho}^{2}(0, \infty)$ of the formal differential operator defined in (1.5).

From the perturbation ansatz $f(y, s)=f_{0}(y)+e^{-\lambda s} w(y)$ it is evident that negative eigenvalues of $\mathcal{A}_{0}$ lead to linear instabilities of $f_{0}$. As a matter of fact, there exists the negative eigenvalue -1 but this is a gauge eigenvalue, i.e., it is related to the freedom of choosing the parameter $T$ in the definition of the self-similar variables and therefore it is not related to an instability of $f_{0}$. Consequently, to prove linear stability of $f_{0}$ it is necessary to rule out the existence of negative eigenvalues of $\mathcal{A}_{0}$ other than -1 . As already mentioned, the most difficult part is to prove the absence of eigenvalues in $(-1,0]$ since this hinges on the particular shape of the potential $V_{0}$. Thus, a rigorous proof of this spectral gap property requires quantitative information on $f_{0}$. Our estimates (1.3) on $f_{0}$ lead to very precise bounds on $V_{0}$ 
and in turn allow us to prove the following stability result. This result is an indispensable ingredient in the proof of the nonlinear asymptotic stability of $f_{0}$ in the companion paper [4].

Theorem 1.6. The only eigenvalue of the operator $\mathcal{A}_{0}$ in the interval $(-\infty, 0]$ is -1 .

Furthermore, the strict bounds on $f_{0}$ could potentially lead to a rigorous treatment of continuation beyond the blow-up, along the lines of the following informal reasoning. In [3] it is conjectured that one can construct a unique continuation for a solution

$$
u_{0}(r, t):=f_{0}\left(\frac{r}{\sqrt{T-t}}\right), \quad t<T
$$

past the blow-up time by defining

$$
u_{0}(r, t):=g_{0}\left(\frac{r}{\sqrt{t-T}}\right), \quad t>T .
$$

In the above definition, $g_{0}$ is a profile of an expanding self-similar solution satisfying

$$
g^{\prime \prime}(y)+\left(\frac{2}{y}+\frac{y}{2}\right) g^{\prime}(y)-\frac{1}{y^{2}} \sin (2 g(y))=0, \quad g(0) \in\{0, \pi\}, \quad g(\infty)=\text { const } .
$$

Note that in the above boundary value problem the expanding profile $g$ can freely select the boundary condition at $y=0$, which means that $u_{0}(0, t)$ may jump from 0 to $\pi$. In effect, the underlying map $F: \mathbb{R}^{3} \rightarrow \mathbb{S}^{3}$ would change its homotopy class in consequence of the blow-up. One of the main motivations for studying the blow-up patterns is to determine whether such a jump occurs.

The formal construction in [3] requires that $g_{0}$ satisfies the following matching condition

$$
g_{0}(\infty)=\lim _{t \rightarrow T+} u_{0}(r, t)=\lim _{t \rightarrow T-} u_{0}(r, t)=f_{0}(\infty) .
$$

That is, the asymptotics of $g_{0}$ and $f_{0}$ have to coincide at infinity. In this sense, the question of unique continuation past the blow-up can be reduced to the question of uniqueness of solutions to the boundary value problem (1.6) with a boundary condition $g_{0}(\infty)=f_{0}(\infty)$. Germain and Rupflin, who studied the boundary value problem (1.6) in [15], prove that the closer $g(\infty)$ is to $\pi / 2$ the more solutions to (1.6) there are. In the more recent paper [14] it is shown that there are at least two stable expanding self-similar solutions with the same initial data, provided $g(\infty)$ is sufficiently close to $\pi / 2$. For $f_{0}$ from Theorem 1.1 one can explicitly compute that $\left|f_{0}(\infty)-\pi / 2\right|>0.56$ which is conjecturally large enough to allow for a unique continuation.

\section{EXISTENCE OF A SELF-SIMILAR SOLUTiON}

The standard approach would be to define $\delta=f-\widetilde{f}_{0}$ and rewrite the equation (1.2) as

$$
\mathcal{L} \delta=\mathcal{R}\left(\widetilde{f}_{0}\right)+\mathcal{N}(\delta)
$$


with

$$
\begin{aligned}
\mathcal{L} \delta(y) & =-\delta^{\prime \prime}(y)-\left(\frac{2}{y}-\frac{y}{2}\right) \delta^{\prime}(y)+\frac{2}{y^{2}} \delta(y)+\widetilde{V}_{0}(y) \delta(y), \quad \widetilde{V}_{0}(y)=\frac{-4 \sin \left(\widetilde{f}_{0}(y)\right)^{2}}{y^{2}} \\
\mathcal{R}\left(\widetilde{f}_{0}\right)(y) & =\widetilde{f}_{0}^{\prime \prime}(y)+\left(\frac{2}{y}-\frac{y}{2}\right) \widetilde{f}_{0}^{\prime}(y)-\frac{1}{y^{2}} \sin \left(2 \widetilde{f}_{0}(y)\right) \\
\mathcal{N}(\delta)(y) & =-\frac{1}{y^{2}}\left(\sin \left(2 \widetilde{f}_{0}(y)+2 \delta(y)\right)-\sin \left(2 \widetilde{f}_{0}(y)\right)-2 \cos \left(2 \widetilde{f}_{0}(y)\right) \delta(y)\right) \\
& =\frac{2}{y^{2}}\left(\cos \left(2 \widetilde{f}_{0}(y)\right) \delta(y)-\cos \left(2 \widetilde{f}_{0}(y)+\delta(y)\right) \sin (\delta(y))\right) .
\end{aligned}
$$

The goal now is to invert the operator $\mathcal{L}$ and prove that

$$
\mathcal{K}(\delta):=\mathcal{L}^{-1}\left(\mathcal{R}\left(\widetilde{f}_{0}\right)+\mathcal{N}(\delta)\right)
$$

is a contraction if $\|\delta\|$ is small enough. Unfortunately this plan cannot succeed as the operator $\mathcal{L}$ contains a very complicated potential $\widetilde{V}_{0}$ : a nonlinear function of $\widetilde{f}_{0}$, which itself is already complicated. Because of the complicated form of the potential, $\mathcal{L}$ cannot be inverted explicitly.

The remedy comes in the form of the following trick. Imagine we can construct an operator $\widetilde{\mathcal{L}}$, which we can invert explicitly, and we rewrite Eq. (2.1) as

$$
\widetilde{\mathcal{L}} \delta=\mathcal{R}\left(\widetilde{f}_{0}\right)+\mathcal{N}(\delta)+(\widetilde{\mathcal{L}}-\mathcal{L}) \delta .
$$

If, in addition, the difference $\widetilde{\mathcal{L}}-\mathcal{L}$ is small in a suitable sense, then the map

$$
\widetilde{\mathcal{K}}(\delta):=\widetilde{\mathcal{L}}^{-1}\left(\mathcal{R}\left(\widetilde{f}_{0}\right)+\mathcal{N}(\delta)+(\widetilde{\mathcal{L}}-\mathcal{L}) \delta\right)
$$

turns out to be the right object to apply a contraction mapping principle to, as we can estimate all the objects on the right hand side explicitly. Because solving (2.2) is equivalent to solving (2.1), it is sufficient to find a fixed point of $\widetilde{\mathcal{K}}$. At this point, it remains to show that $\widetilde{\mathcal{K}}$ is a contraction on a suitable closed subset of a Banach space, chosen here as

$$
X=\left\{\delta \in C^{1}([0, \infty)),\|\delta\| \leq 5 \cdot 10^{-4}\right\}
$$

with $\|\cdot\|$ given in Theorem 1.1. The rest of the proof is divided as follows, where we abbreviate $\|\cdot\|_{\infty}:=\|\cdot\|_{L^{\infty}(0, \infty)}$.

(1) We construct an operator $\widetilde{\mathcal{L}}$ so that

$$
\left\|\widetilde{\mathcal{L}}^{-1} \alpha\right\| \leq c_{\mathcal{L}}\left\|p_{2} \alpha\right\|_{\infty} \quad p_{2}(y)=\frac{\left(2+y^{2}\right)^{5 / 2}}{3 \sqrt{2} y\left(4+y^{2}\right)}
$$

for $c_{\mathcal{L}}=180$ and any function $\alpha$ with a finite $\left\|p_{2} \alpha\right\|_{\infty}$. In addition, for the constructed operator $\widetilde{\mathcal{L}}$, the difference $\widetilde{\mathcal{L}}-\mathcal{L}$ is small (see the estimate in the next point).

(2) We show that for $\delta, \gamma \in X$

$$
\begin{aligned}
&\left\|p_{2} \mathcal{R}\left(\widetilde{f}_{0}\right)\right\|_{\infty} \leq c_{\mathcal{R}} \\
&\left\|p_{2} \mathcal{N}(\delta)\right\|_{\infty} \leq c_{\mathcal{N}}\|\delta\|^{2} \\
&\left\|p_{2}(\mathcal{N}(\delta)-\mathcal{N}(\gamma))\right\|_{\infty} \leq c_{\mathcal{N}}(\|\delta\|+\|\gamma\|)\|\delta-\gamma\| \\
&\left\|p_{2}(\widetilde{\mathcal{L}}-\mathcal{L}) \delta\right\|_{\infty} \leq c_{\widetilde{\mathcal{L}}}\|\delta\| \\
& 6
\end{aligned}
$$


with constants $c_{\mathcal{R}}=10^{-6}, c_{\mathcal{N}}=4$ and $c_{\widetilde{\mathcal{L}}}=4 \cdot 10^{-5}$

(3) Combining these results we prove that $\widetilde{\mathcal{K}}$ has a unique fixed point in $X$. This follows immediately from the estimates from points (1) and (2) and from the contraction mapping principle. Indeed, for all $\delta, \gamma \in X$ we have

$$
\begin{aligned}
\|\widetilde{\mathcal{K}}(\delta)\| & \leq c_{\mathcal{L}}\left(c_{\mathcal{R}}+c_{\widetilde{\mathcal{L}}}\|\delta\|+c_{\mathcal{N}}\|\delta\|^{2}\right) \\
& \leq 180\left(10^{-6}+\left(4 \cdot 10^{-5}\right) \cdot\left(5 \cdot 10^{-4}\right)+4 \cdot\left(5 \cdot 10^{-4}\right)^{2}\right) \\
& =3.636 \cdot 10^{-4}<5 \cdot 10^{-4}
\end{aligned}
$$

so $\widetilde{\mathcal{K}}$ maps back into $X$, and $\widetilde{\mathcal{K}}$ is a contraction because

$$
\begin{aligned}
\|\widetilde{\mathcal{K}}(\delta)-\widetilde{\mathcal{K}}(\gamma)\| & =\left\|\widetilde{\mathcal{L}}^{-1}(\mathcal{N}(\delta)-\mathcal{N}(\gamma)+(\widetilde{\mathcal{L}}-\mathcal{L})(\delta-\gamma))\right\| \\
& \leq c_{\mathcal{L}}\left(c_{\mathcal{N}}(\|\delta\|+\|\gamma\|)+c_{\widetilde{\mathcal{L}}}\right)\|\delta-\gamma\| \\
& \leq 180\left(4 \cdot 10^{-3}+4 \cdot 10^{-5}\right)\|\delta-\gamma\| \\
& =0.7272\|\delta-\gamma\| .
\end{aligned}
$$

Consequently, by the contraction mapping principle and elementary regularity theory there exists a $\delta_{0} \in X \cap C^{\infty}([0, \infty))$ that solves (2.2). But such a $\delta_{0}$ must also solve (2.1) and thus, $f_{0}:=\widetilde{f}_{0}+\delta$ must solve (1.2) and $f_{0} \in C^{\infty}([0, \infty))$.

Remark 2.1. The main difficulty in the above procedure is to determine the approximate solution $\widetilde{f}_{0}$ and the operator $\widetilde{\mathcal{L}}$ such that the constants $c_{\mathcal{R}}$ and $c_{\widetilde{\mathcal{L}}}$ are small enough for $\widetilde{\mathcal{K}}$ to be a contraction. In contrast, we do not have much influence on the constants $c_{\mathcal{N}}$ and $c_{\mathcal{L}}$; they are the constants of our problem.

Remark 2.2. Because of the complicated form of the approximations $\tilde{f}_{0}$ and $\widetilde{\mathcal{L}}$, our proof relies on computer algebra and rigorous computer-assisted methods for estimating rational functions (namely the method of interval arithmetic). These methods will be described in detail in the following sections.

2.1. Estimate for the remainder term $\mathcal{R}\left(\widetilde{f}_{0}\right)$. The remainder term is the simplest one to analyze so we shall use it to demonstrate the method of interval arithmetic, which we shall use extensively throughout the paper. We defined the remainder term as

$$
\mathcal{R}\left(\widetilde{f}_{0}\right)=\widetilde{f}_{0}^{\prime \prime}+\left(\frac{2}{y}-\frac{y}{2}\right) \widetilde{f}_{0}^{\prime}-\frac{1}{y^{2}} \sin \left(2 \widetilde{f}_{0}\right)
$$

with $\widetilde{f}_{0}$ given explicitly as

$$
\widetilde{f}_{0}(y)=2 \arctan \left(g_{0}(y)\right), \quad g_{0}(y)=\sum_{n=0}^{14}\left(f_{0}\right)_{n} T_{2 n+1}\left(\frac{y}{\sqrt{2+y^{2}}}\right) .
$$

Now we argue that the remainder term, multiplied by the weight $p_{2}$, is a rational function of $y$ (we will need this fact later on to apply the interval arithmetic bounds). The main difficulty lies in convincing oneself that the square root in the definition of $g_{0}$ eventually does not show up in the expression $p_{2} \mathcal{R}\left(\widetilde{f}_{0}\right)$.

Let us start by writing the remainder term in terms of $g_{0}$

$$
\mathcal{R}\left(\widetilde{f}_{0}\right)=\frac{2}{1+g_{0}^{2}}\left(g_{0}^{\prime \prime}+\left(\frac{2}{y}-\frac{y}{2}-\frac{2 g_{0} g_{0}^{\prime}}{1+g_{0}^{2}}\right) g_{0}^{\prime}-\frac{2 g_{0}\left(1-g_{0}^{2}\right)}{y^{2}\left(1+g_{0}^{2}\right)}\right) .
$$


Now, because $T_{2 n+1}(x)=x P_{n}\left(x^{2}\right)$, with $P_{n}$ being some polynomial of order $n$, we can write the function $g_{0}$ as $g_{0}(y)=\frac{y}{\sqrt{2+y^{2}}} Q\left(y^{2} /\left(2+y^{2}\right)\right.$ ) (with some polynomial $Q$ ). Consequently, we factored out the square root. It is now straightforward to see that the square root can be eventually factored out of $\mathcal{R}\left(\widetilde{f}_{0}\right)$ in a similar fashion. Going back to the definition of the weight $p_{2}$ we obtain the representation

$$
p_{2} \mathcal{R}\left(\widetilde{f}_{0}\right)(y)=\frac{U\left(y^{2} /\left(2+y^{2}\right)\right)}{V\left(y^{2} /\left(2+y^{2}\right)\right)}
$$

where $U$ and $V$ are polynomials with rational coefficients. As the last step, we compactify the domain to the interval $[0,1]$ by replacing $y \in[0, \infty)$ with $x=y^{2} /\left(2+y^{2}\right) \in[0,1]$. We refrain from writing down the polynomials $U$ and $V$ explicitly as they are of order 44 and 59 , respectively, with large integer coefficients.

We now run into the core of the problem: how to estimate such a complicated rational function? Depending on how rigorous we want to be, such an estimate might be straightforward and produced by simply plotting the graph of the function or incredibly difficult if we decide to work on the rational function directly and show the bound explicitly. We decided on an approach that is almost as simple as plotting the function but still rigorous: interval arithmetic.

Interval arithmetic is essentially a way to find bounds on the range of a function on a given interval. Say we are interested in estimating the range of the function $f(x)=x-x^{2}$ on an interval $x \in[0,1]$ (naturally, one can do this explicitly but the point is to illustrate the method). In the interval arithmetic approach we first compute the range of $x$, which is $[0,1]$, then we compute the range of $-x^{2}$, which is $[-1,0]$. Now the key observation is that the range of a sum has to be contained in the sum of the ranges (defined as $[a, b]+[c, d]:=[a+c, b+d]$ ), that is

$$
f(x) \in[0,1]+[-1,0]=[-1,-1], \quad x \in[0,1] .
$$

Indeed, one finds that the exact range of the function $f$ is $[0,1 / 4] \subset[-1,1]$.

One can define the remaining operations on intervals as follows.

Definition 2.3 (Interval arithmetic). For $a, b, c, d \in \mathbb{R}$ we define

$$
\begin{aligned}
{[a, b]+[c, d] } & :=[a+c, b+d] \\
{[a, b]-[c, d] } & :=[a-d, b-c] \\
{[a, b] \cdot[c, d] } & :=[\min \{a c, a d, b c, b d\}, \max \{a c, a d, b c, b d\}] \\
\frac{[a, b]}{[c, d]} & :=[a, b] \cdot\left[\frac{1}{d}, \frac{1}{c}\right], \quad 0 \notin[c, d]
\end{aligned}
$$

(here we assume that the intervals on the left hand side are nonempty, so that $a \leq b$ and $c \leq d)$. Moreover, the operations mixing intervals and real numbers can be included by interpreting $a \in \mathbb{R}$ as $[a, a]$.

It is straightforward to check that these definitions lead to the following statement.

Theorem 2.4. Let $*$ be any of the operations defined in 2.3 and let $x \in[a, b]$ and $y \in[c, d]$. Then we have $x * y \in[a, b] *[c, d]$. 
This theorem allows us to extend the operations on numbers, like in our example $x-x^{2}$, to operations on sets. Consequently, $f(x)=x-x^{2}$ can be interpreted as either a function on real numbers giving $f(1)=0$, or a function on intervals giving $f([0,1])=[-1,1]$. Thanks to Theorem 2.4, for every $x \in[0,1]$, we have $f(x) \in f([0,1])=[-1,1]$.

There are, however, some pitfalls one should be aware of when using interval arithmetic. The first problem (also called the dependency problem) is that the resulting bound strongly depends on the algebraic form of the expression. For example, if we write $f(x)$ as $x(1-x)$ the bound becomes $f([0,1])=[0,1]$, so rewriting the expression might improve or degrade the estimate; the resulting bound will still be rigorous but it may simply be less efficient. Next, in most cases a single interval is insufficient to obtain a satisfactory estimate and splitting the interval into two or more subintervals will often improve the result. For example we can write the interval $[0,1]$ as $[0,1 / 2] \cup[1 / 2,1]$, effectively splitting the domain into two parts; so for $x \in[0,1]$ we have $f(x)=x(1-x) \in f([0,1 / 2]) \cup f([1 / 2,1])=[0,1 / 2] \cup[0,1 / 2]=[0,1 / 2]$, which is closer to the optimal estimate. For a comprehensive summary of these and other aspects of interval arithmetic methods, the reader is referred to [23] or [24].

We remove the ambiguity coming from the dependency problem by writing each rational function $R$, which we want to estimate on the domain $x \in[0,1]$, in so-called Bernstein form

$$
R(x)=\frac{\sum_{i=0}^{n} a_{i} x^{i}(1-x)^{n-i}}{\sum_{j=0}^{m} b_{j} x^{j}(1-x)^{m-j}}
$$

where $a_{i}, b_{j} \in \mathbb{Q}$. This representation is unique provided the fraction is reduced. The Bernstein form seems to improve the estimates coming from interval arithmetic (note that writing $f(x)$ from our example as $x(1-x)$ is actually rewriting it in Bernstein form). Another benefit of the Bernstein form is that it is trivial to see if the denominator of $R$ is strictly positive by simply checking if all the coefficients $b_{j}$ are nonnegative (still, at least one has to be positive).

Then, we mince the domain of our function by bisecting each interval $[a, b]$, for which the interval $R([a, b])$ turned out to be too broad, and then we take a union of the resulting estimates. Specifically, we rewrite the offending interval as $[a, b]=[a,(a+b) / 2] \cup[(a+b) / 2, b]$ and we use $R([a, b]) \subset R([a,(a+b) / 2]) \cup R([(a+b) / 2, b])$.

Unfortunately, the rational functions we are dealing with are too complex and the number of subintervals too large to perform all the computations by hand or even explicitly include them in this paper. However, by following the procedure just explained, it is straightforward to verify our claims using any suitable software package. In addition, there exists an on-line supplement to this article which consists of a Mathematica Notebook that contains all the computations. Also, having discussed the method in detail here, in the rest of this paper we shall use the interval arithmetic freely.

Running the described algorithm we get

$$
\left\|p_{2} \mathcal{R}\left(\widetilde{f}_{0}\right)\right\|_{\infty} \leq c_{\mathcal{R}}, \quad c_{\mathcal{R}}=10^{-6} .
$$

2.2. Estimates for the nonlinear term $\mathcal{N}(\delta)$. The nonlinear term $\mathcal{N}(\delta)$ was defined as

$$
\begin{aligned}
\mathcal{N}(\delta) & =\frac{1}{y^{2}}\left(2 \cos \left(2 \widetilde{f}_{0}\right) \delta-2 \cos \left(2 \widetilde{f}_{0}+\delta\right) \sin (\delta)\right) \\
& =\frac{2}{y^{2}}\left(\cos \left(2 \widetilde{f}_{0}\right)(\delta-\sin (\delta))+4 \cos (\delta / 2) \sin \left(2 \widetilde{f}_{0}+\delta / 2\right) \sin ^{2}(\delta / 2)\right)
\end{aligned}
$$


so that

$$
|\mathcal{N}(\delta)| \leq \frac{2}{y^{2}}\left(\frac{1}{6}|\delta|^{3}+|\delta|^{2}\left(\left|\sin \left(2 \widetilde{f}_{0}\right)\right|+\frac{1}{2}|\delta|\right)\right)=\frac{2}{y^{2}}|\delta|^{2}\left(\left|\sin \left(2 \widetilde{f}_{0}\right)\right|+\frac{2}{3}|\delta|\right) .
$$

This leads to

$$
\left|p_{2} \mathcal{N}(\delta)\right| \leq\left(p_{1} \delta\right)^{2}\left(\left|\frac{2 p_{2}}{p_{1}^{2} y^{2}} \sin \left(2 \widetilde{f}_{0}\right)\right|+\frac{4 p_{2}}{3 p_{1}^{3} y^{2}}\left|p_{1} \delta\right|\right)
$$

and now, according to the previous section, interval arithmetic provides the following bound on the first term,

$$
\left|\frac{2 p_{2}}{p_{1}^{2} y^{2}} \sin \left(2 \widetilde{f}_{0}\right)\right| \leq 3.9
$$

while the second term can be bounded explicitly using the definitions of the weights $p_{1}, p_{2}$ and $p_{3}$,

$$
\frac{4 p_{2}}{3 p_{1}^{3} y^{2}}=\frac{8\left(2+y^{2}\right)}{9\left(4+y^{2}\right)} \leq 1
$$

In summary, we obtain

$$
\left\|p_{2} \mathcal{N}(\delta)\right\|_{\infty} \leq\|\delta\|^{2}(3.9+\|\delta\|) .
$$

Now, if $\delta \in X$, we have $\|\delta\| \leq 5 \cdot 10^{-4}$ so that

$$
\left\|p_{2} \mathcal{N}(\delta)\right\|_{\infty} \leq c_{\mathcal{N}}\|\delta\|^{2}, \quad c_{\mathcal{N}}=4 .
$$

A similar reformulation can be carried out for the difference

$$
\begin{aligned}
\mathcal{N}(\delta)-\mathcal{N}(\gamma)= & \frac{2}{y^{2}}\left(\cos \left(2 \widetilde{f}_{0}\right)(\delta-\gamma)-\cos \left(2 \widetilde{f}_{0}+\delta\right) \sin (\delta)+\cos \left(2 \widetilde{f}_{0}+\gamma\right) \sin (\gamma)\right) \\
= & \frac{2}{y^{2}}\left(2 \sin ((\delta+\gamma) / 2) \sin \left(2 \widetilde{f}_{0}+(\delta+\gamma) / 2\right) \sin (\delta-\gamma)\right. \\
& \left.+\cos \left(2 \widetilde{f}_{0}\right)((\delta-\gamma)-\sin (\delta-\gamma))\right),
\end{aligned}
$$

Given the new form of the difference we can apply essentially the same estimates as for the term $\mathcal{N}(\delta)$ leading to

$$
\left|p_{2}(\mathcal{N}(\delta)-\mathcal{N}(\gamma))\right| \leq\left|p_{1}(\delta-\gamma)\right|\left(\left|p_{1} \delta\right|+\left|p_{1} \gamma\right|\right)\left(\frac{2 p_{2}}{y^{2} p_{1}^{2}}\left|\sin \left(2 \widetilde{f}_{0}\right)\right|+\frac{4 p_{2}}{3 y^{2} p_{1}^{3}}\left(\left|p_{1} \delta\right|+\left|p_{1} \gamma\right|\right)\right)
$$

and then, again using (2.4) and (2.5), to

$$
\left\|p_{2}(\mathcal{N}(\delta)-\mathcal{N}(\gamma))\right\|_{\infty} \leq\|\delta-\gamma\|(\|\delta\|+\|\gamma\|)(3.9+\|\delta\|+\|\gamma\|) .
$$

But for $\delta, \gamma \in X$ we have $\|\delta\|+\|\gamma\| \leq 2 \cdot 5 \cdot 10^{-4}$ so

$$
\left\|p_{2}(\mathcal{N}(\delta)-\mathcal{N}(\gamma))\right\|_{\infty} \leq c_{\mathcal{N}}\|\delta-\gamma\|(\|\delta\|+\|\gamma\|) .
$$


2.3. The approximate operator $\widetilde{\mathcal{L}}$. We can write an inverse of $\mathcal{L}$ as

$$
\mathcal{L}^{-1} \delta(y)=\int_{0}^{\infty} G(x, y) \delta(x) d x
$$

with the Green's function defined as

$$
G(x, y)=\frac{-1}{W\left(w_{0}, w_{1}\right)(x)} \begin{cases}w_{0}(y) w_{1}(x) & y \leq x \\ w_{0}(x) w_{1}(y) & x \leq y\end{cases}
$$

where $W\left(w_{0}, w_{1}\right)=w_{0} w_{1}^{\prime}-w_{0}^{\prime} w_{1}$. The functions $w_{0}$ and $w_{1}$ satisfy the differential equation

$$
\mathcal{L} w_{0}=\mathcal{L} w_{1}=0
$$

with boundary conditions

$$
\begin{aligned}
& w_{0}(y)=y+\mathcal{O}\left(y^{3}\right), \\
& y \rightarrow 0 \\
& w_{1}(y)=1+\mathcal{O}\left(y^{-2}\right), \\
& y \rightarrow \infty \text {. }
\end{aligned}
$$

Naturally, these fundamental solutions are unknown in closed form.

Assume though, that we can find a pair of approximate solutions $\widetilde{w}_{0} \approx w_{0}$ and $\widetilde{w}_{1} \approx w_{1}$ with the same boundary conditions as the exact solutions. Then the solutions $\left(\widetilde{w}_{0}, \widetilde{w}_{1}\right)$ uniquely determine an operator $\widetilde{\mathcal{L}}=\mathcal{L}+P \partial_{y}+Q$ by demanding that

$$
\widetilde{\mathcal{L}} \widetilde{w}_{0}=\widetilde{\mathcal{L}} \widetilde{w}_{1}=0 \text {. }
$$

Under such conditions, the coefficients $P$ and $Q$ are given by

$$
\begin{aligned}
& P=\frac{-1}{W\left(\widetilde{w}_{0}, \widetilde{w}_{1}\right)}\left(\mathcal{L} \widetilde{w}_{1} \widetilde{w}_{0}-\mathcal{L} \widetilde{w}_{0} \widetilde{w}_{1}\right), \\
& Q=\frac{1}{W\left(\widetilde{w}_{0}, \widetilde{w}_{1}\right)}\left(\mathcal{L} \widetilde{w}_{1} \widetilde{w}_{0}^{\prime}-\mathcal{L} \widetilde{w}_{0} \widetilde{w}_{1}^{\prime}\right) .
\end{aligned}
$$

With $\widetilde{w}_{0}$ and $\widetilde{w}_{1}$ known explicitly, the operator $\widetilde{\mathcal{L}}$ has a closed form Green's function

$$
\widetilde{G}(x, y)=\frac{-1}{W\left(\widetilde{w}_{0}, \widetilde{w}_{1}\right)(x)}\left\{\begin{array}{ll}
\widetilde{w}_{0}(y) \widetilde{w}_{1}(x) & y \leq x \\
\widetilde{w}_{0}(x) \widetilde{w}_{1}(y) & x \leq y
\end{array} .\right.
$$

Now let us compute a robust, even if slightly inefficient, estimate on $c_{\mathcal{L}}$. For that we are going to need the free part $\mathcal{L}_{0}$ of the operator $\mathcal{L}$, defined as

$$
\mathcal{L}_{0} \delta(y)=-\delta^{\prime \prime}(y)-\left(\frac{2}{y}-\frac{y}{2}\right) \delta^{\prime}(y)+\frac{2}{y^{2}} \delta(y)
$$

which is simply $\mathcal{L}$ without the potential term $\widetilde{V}_{0}$. Let us denote the corresponding free Green's function by

$$
G_{0}(x, y)=\frac{-1}{W\left(v_{0}, v_{1}\right)(x)}\left\{\begin{array}{ll}
v_{0}(y) v_{1}(x) & y \leq x \\
v_{0}(x) v_{1}(y) & x \leq y
\end{array},\right.
$$

where $v_{0}$ and $v_{1}$ are fundamental solutions of $\mathcal{L}_{0}$ with the same boundary behavior as $w_{0}$ and $w_{1}$ (condition (2.6) ). In fact, these solutions are known explicitly,

$$
v_{0}(y)=\frac{3}{y^{2}} e^{y^{2} / 4}\left(-y+\left(2+y^{2}\right) D_{+}(y / 2)\right), \quad v_{1}(y)=1+\frac{2}{y^{2}}
$$


where $D_{+}$is the Dawson integral

$$
D_{+}(y)=e^{-y^{2}} \int_{0}^{y} e^{x^{2}} d x .
$$

It is not hard to see that $v_{0}, v_{1}>0$ on $(0, \infty)$ and the Wrońskian is simply

$$
\frac{-1}{W\left(v_{0}, v_{1}\right)(x)}=\frac{1}{6} x^{2} e^{-\frac{x^{2}}{4}} \text {. }
$$

Now we are ready to take a closer look at the inverse of $\widetilde{\mathcal{L}}$. We start by rewriting

$$
\begin{aligned}
p_{1}(y) \widetilde{\mathcal{L}}^{-1} \alpha(y) & =\int_{0}^{\infty} \widetilde{G}(x, y) p_{1}(y) \alpha(x) d x \\
& =\int_{0}^{\infty}\left(G_{0}(x, y) \frac{p_{1}(y)}{p_{2}(x)}\right)\left(\frac{\widetilde{G}(x, y)}{G_{0}(x, y)}\right) p_{2}(x) \alpha(x) d x .
\end{aligned}
$$

For convenience let us denote the ratio $\widetilde{G} / G_{0}$ as

$$
H(x, y):=\frac{\widetilde{G}(x, y)}{G_{0}(x, y)} .
$$

Taking the $L^{\infty}(0, \infty)$ norm of (2.9), and pulling some terms out of the integral, we get

$$
\begin{aligned}
\left\|p_{1} \widetilde{\mathcal{L}}^{-1} \alpha\right\|_{\infty} & \leq \sup _{y>0}\left(\int_{0}^{\infty}\left|G_{0}(x, y)\right| \frac{p_{1}(y)}{p_{2}(x)} d x\right)\|H\|_{\infty}\left\|p_{2} \alpha\right\|_{\infty} \\
& =\sup _{y>0}\left(\int_{0}^{\infty} G_{0}(x, y) \frac{p_{1}(y)}{p_{2}(x)} d x\right)\|H\|_{\infty}\left\|p_{2} \alpha\right\|_{\infty},
\end{aligned}
$$

where we abbreviate $\|H\|_{\infty}:=\sup _{x, y>0}|H(x, y)|$. In the last equality we dropped the absolute value using the positivity of $G_{0}$. By our choice of the weights $p_{1}$ and $p_{2}$ (this is actually their defining property), we have $\mathcal{L}_{0} \frac{1}{p_{1}}=\frac{1}{p_{2}}$ or, equivalently,

$$
\int_{0}^{\infty} G_{0}(x, y) \frac{p_{1}(y)}{p_{2}(x)} d x=1
$$

hence,

$$
\left\|p_{1} \widetilde{\mathcal{L}}^{-1} \alpha\right\|_{\infty} \leq\|H\|_{\infty}\left\|p_{2} \alpha\right\|_{\infty} .
$$

Similarly, for the derivative we have

$$
p_{3}(y)\left(\widetilde{\mathcal{L}}^{-1} \alpha\right)^{\prime}(y)=\int_{0}^{\infty}\left(\partial_{y} G_{0}(x, y) \frac{p_{3}(y)}{p_{2}(x)}\right) H_{1}(x, y) p_{2}(x) \alpha(x) d x,
$$

where

$$
H_{1}(x, y):=\frac{\partial_{y} \widetilde{G}(x, y)}{\partial_{y} G_{0}(x, y)} .
$$

To estimate the above integral, we start with the following trick: we first take a derivative of (2.10) after dividing it by $p_{1}$ to get

$$
\int_{0}^{\infty} \partial_{y} G_{0}(x, y) \frac{p_{3}(y)}{p_{2}(x)} d x=1, \quad \frac{1}{p_{3}(y)}=\left(\frac{1}{p_{1}}\right)^{\prime}(y)=\frac{2 \sqrt{2}}{\left(2+y^{2}\right)^{3 / 2}} .
$$


The formula on the right above is, in fact, the defining property of the weight $p_{3}$. Unfortunately we cannot use the elegant formula (2.12) directly, as we did with (2.10), because $\partial_{y} G_{0}(x, y)$ changes sign on the diagonal $\{(x, y): x=y\}$. Indeed, if we go back to the definitions of fundamental solutions we see that $v_{0}^{\prime}(y)=12 e^{y^{2} / 4} y^{-3}\left(y / 2-D_{+}(y / 2)\right)$ is positive whereas $v_{1}^{\prime}(y)=-4 / y^{3}$ is negative. Consequently, we cannot remove the absolute value in the estimate

$$
\left\|p_{3}\left(\widetilde{\mathcal{L}}^{-1} \alpha\right)^{\prime}\right\|_{\infty} \leq \sup _{y>0}\left(\int_{0}^{\infty}\left|\partial_{y} G_{0}(x, y) \frac{p_{3}(y)}{p_{2}(x)}\right| d x\right)\left\|H_{1}\right\|_{\infty}\left\|p_{2} \alpha\right\|_{\infty} .
$$

Thus, we have to take a brief detour to deal with the integral

$$
\int_{0}^{\infty}\left|\partial_{y} G_{0}(x, y) \frac{p_{3}(y)}{p_{2}(x)}\right| d x
$$

Luckily, this integral can be computed explicitly. To see this, we use the definition (2.7) of the Green's function to split (2.13) into two integrals

$$
\begin{aligned}
\int_{0}^{\infty} \mid \partial_{y} G_{0}(x, y) \frac{p_{3}(y)}{p_{2}(x) \mid} d x & =-\frac{1}{6} \int_{0}^{y} x^{2} e^{-\frac{x^{2}}{4}} v_{1}^{\prime}(y) v_{0}(x) \frac{p_{3}(y)}{p_{2}(x)} d x \\
& +\frac{1}{6} \int_{y}^{\infty} x^{2} e^{-\frac{x^{2}}{4}} v_{1}(x) v_{0}^{\prime}(y) \frac{p_{3}(y)}{p_{2}(x)} d x \\
& =: I_{0}(y)+I_{1}(y)
\end{aligned}
$$

Above we also used the fact that $v_{0}, v_{0}^{\prime}$ and $v_{1}$, as well as the weights $p_{2}$ and $p_{3}$, are all nonnegative and that $v_{1}^{\prime}$ is negative. The integral $I_{1}(y)$ can be computed explicitly,

$$
\begin{aligned}
I_{1}(y) & =\frac{1}{6} v_{0}^{\prime}(y) p_{3}(y) \int_{y}^{\infty} x^{2} e^{-\frac{x^{2}}{4}} v_{1}(x) \frac{1}{p_{2}(x)} d x \\
& =\frac{1}{\sqrt{2}} v_{0}^{\prime}(y) p_{3}(y) \int_{y}^{\infty} \frac{x\left(4+x^{2}\right)}{\left(2+x^{2}\right)^{3 / 2}} e^{-\frac{x^{2}}{4}} d x \\
& =\sqrt{2} v_{0}^{\prime}(y) p_{3}(y) \frac{e^{-\frac{y^{2}}{4}}}{\sqrt{2+y^{2}}} .
\end{aligned}
$$

By definition, $p_{3}(y)=\left(2+y^{2}\right)^{3 / 2} /(2 \sqrt{2})$ and thus, we arrive at the final formula

$$
I_{1}(y)=6 y^{-3}\left(2+y^{2}\right)\left(y / 2-D_{+}(y / 2)\right)=3-2 y^{-1} e^{-\frac{y^{2}}{4}} v_{0}(y) .
$$

In the last equality we used the definition of $v_{0}$ to replace $D_{+}$with $v_{0}$.

To compute $I_{0}$, we go back to (2.12) and observe that

$$
1=\int_{0}^{\infty} \partial_{y} G_{0}(x, y) \frac{p_{3}(y)}{p_{2}(x)} d x=-I_{0}(y)+I_{1}(y)
$$

Consequently, $I_{0}(y)=I_{1}(y)-1$ and thus,

$$
\int_{0}^{\infty}\left|\partial_{y} G_{0}(x, y) \frac{p_{3}(y)}{p_{2}(x)}\right| d x=I_{0}(y)+I_{1}(y)=2 I_{1}(y)-1=5-4 y^{-1} e^{-\frac{y^{2}}{4}} v_{0}(y) \leq 5 .
$$

This yields

$$
\left\|p_{3}\left(\widetilde{\mathcal{L}}^{-1} \alpha\right)^{\prime}\right\|_{\infty} \leq 5\left\|H_{1}\right\|_{\infty}\left\|p_{2} \alpha\right\|_{\infty}
$$


and thus,

$$
\begin{aligned}
\left\|\widetilde{\mathcal{L}}^{-1} \alpha\right\| & =\left\|p_{1} \widetilde{\mathcal{L}}^{-1} \alpha\right\|_{\infty}+\left\|p_{3}\left(\widetilde{\mathcal{L}}^{-1} \alpha\right)^{\prime}\right\|_{\infty} \\
& \leq\left(\|H\|_{\infty}+5\left\|H_{1}\right\|_{\infty}\right)\left\|p_{2} \alpha\right\|_{\infty} .
\end{aligned}
$$

To estimate $\|H\|_{\infty}$, we write

$$
H(x, y)=\frac{W\left(v_{0}, v_{1}\right)(x)}{W\left(\widetilde{w}_{0}, \widetilde{w}_{1}\right)(x)} \begin{cases}h_{0}(y) h_{1}(x) & y \leq x \\ h_{0}(x) h_{1}(y) & x \leq y\end{cases}
$$

where

$$
h_{0}(x)=\frac{\widetilde{w}_{0}(x)}{v_{0}(x)}, \quad h_{1}(x)=\frac{\widetilde{w}_{1}(x)}{v_{1}(x)} .
$$

At this point it remains to pick a particular pair $\left(\widetilde{w}_{0}, \widetilde{w}_{1}\right)$, approximating $\left(w_{0}, w_{1}\right)$, which leads to satisfactory estimates on $\widetilde{\mathcal{L}}-\mathcal{L}$ (see section 2.4 below). Our numerically inspired guess is

$$
\begin{aligned}
& \widetilde{w}_{0}(y):=v_{0}(y) \sum_{n=0}^{44}\left(w_{0}\right)_{n} T_{2 n}\left(\frac{y}{\sqrt{y^{2}+4}}\right) \\
& \widetilde{w}_{1}(y):=v_{1}(y) \sum_{n=0}^{37}\left(w_{1}\right)_{n} T_{n}\left(\frac{y-2}{y+2}\right)
\end{aligned}
$$

with the coefficients $\left(w_{0}\right)_{n}$ and $\left(w_{1}\right)_{n}$ presented in Tables B.2 and B.3 respectively. A cautious reader will notice that the definition (2.15) is not complete: the last two coefficients in (2.15), $\left(w_{1}\right)_{36}$ and $\left(w_{1}\right)_{37}$, are missing from Table B.3. To remove this ambiguity we impose two additional conditions that fix the last two coefficients; we demand that

$$
\left.\frac{d}{d y} \frac{\widetilde{w}_{1}(y)}{v_{1}(y)}\right|_{y=0}=0,\left.\quad \frac{d}{d y} \frac{\widetilde{w}_{1}(y)}{v_{1}(y)}\right|_{y=\infty}=0 .
$$

These two conditions ensure that $\widetilde{w}_{1}$ has the correct endpoint behavior. Both coefficients $\left(w_{1}\right)_{36}$ and $\left(w_{1}\right)_{37}$ contribute only a correction of order $10^{-12}$ to the whole sum.

We have $\left\|h_{j}\right\|_{\infty} \leq 1.01$ for $j=0,1$ (see the appendix), so

$$
\|H\|_{\infty} \leq 1.01^{2}\left\|\frac{W\left(v_{0}, v_{1}\right)}{W\left(\widetilde{w}_{0}, \widetilde{w}_{1}\right)}\right\|_{\infty} .
$$

The contents of the norm on the right hand side is a function of a single variable and thus it can be easily estimated by the method of interval arithmetic. After performing the computations we arrive at

$$
\|H\|_{\infty} \leq 1.01^{2} \cdot 28<30 .
$$

The function $H_{1}$ can be estimated in a similar fashion. Indeed, we have

$$
H_{1}(x, y)=\frac{W\left(v_{0}, v_{1}\right)(x)}{W\left(\widetilde{w}_{0}, \widetilde{w}_{1}\right)(x)} \begin{cases}\frac{\widetilde{w}_{0}^{\prime}(y)}{v_{0}^{\prime}(y)} h_{1}(x) & y \leq x \\ h_{0}(x) \frac{\widetilde{w}_{1}^{\prime}(y)}{v_{1}^{\prime}(y)} & x<y\end{cases}
$$


Again, by interval arithmetic, we show that $\left\|\widetilde{w}_{j}^{\prime} / v_{j}^{\prime}\right\|_{\infty} \leq 1.01$ for $j=0,1$ and then the same estimate as for $\|H\|_{\infty}$ follows,

$$
\left\|H_{1}\right\|_{\infty} \leq 1.01^{2} \cdot 28<30 .
$$

Putting everything together, we arrive at

$$
\left\|\widetilde{\mathcal{L}}^{-1} \alpha\right\| \leq\left(\|H\|_{\infty}+5\left\|H_{1}\right\|_{\infty}\right)\left\|p_{2} \alpha\right\|_{\infty} \leq c_{\mathcal{L}}\left\|p_{2} \alpha\right\|_{\infty}, \quad c_{\mathcal{L}}:=(1+5) \cdot 30=180 .
$$

2.4. Linear part and the constant $c_{\tilde{\mathcal{L}}}$. We have already constructed the operator $\widetilde{\mathcal{L}}$ and its inverse, now it remains to show that the difference $\widetilde{\mathcal{L}}-\mathcal{L}$ is small. To this end we estimate

$$
p_{2}(\widetilde{\mathcal{L}}-\mathcal{L}) \delta=p_{2}\left(P \delta^{\prime}+Q \delta\right)
$$

where $Q$ and $P$ were given in the previous section as

$$
\begin{aligned}
& P=\frac{-1}{W\left(\widetilde{w}_{0}, \widetilde{w}_{1}\right)}\left(\mathcal{L} \widetilde{w}_{1} \widetilde{w}_{0}-\mathcal{L} \widetilde{w}_{0} \widetilde{w}_{1}\right), \\
& Q=\frac{1}{W\left(\widetilde{w}_{0}, \widetilde{w}_{1}\right)}\left(\mathcal{L} \widetilde{w}_{1} \widetilde{w}_{0}^{\prime}-\mathcal{L} \widetilde{w}_{0} \widetilde{w}_{1}^{\prime}\right),
\end{aligned}
$$

with $\widetilde{w}_{0}$ and $\widetilde{w}_{1}$ defined in (2.14) and (2.15), respectively. Note that (2.16) implies

$$
\begin{aligned}
\left\|p_{2}(\widetilde{\mathcal{L}}-\mathcal{L}) \delta\right\|_{\infty} & \leq\left(\left\|\frac{p_{2}}{p_{3}} P\right\|_{\infty}\left\|p_{3} \delta^{\prime}\right\|_{\infty}+\left\|\frac{p_{2}}{p_{1}} Q\right\|_{\infty}\left\|p_{1} \delta\right\|_{\infty}\right) \\
& \leq\left(\left\|\frac{p_{2}}{p_{3}} P\right\|_{\infty}+\left\|\frac{p_{2}}{p_{1}} Q\right\|_{\infty}\right)\|\delta\|,
\end{aligned}
$$

so it is enough to estimate each of the two terms in the big parenthesis. With the help of interval arithmetic we find that they obey

$$
\begin{aligned}
& \left\|\frac{p_{2}}{p_{3}} P\right\|_{\infty} \leq c_{P}=2 \cdot 10^{-5}, \\
& \left\|\frac{p_{2}}{p_{1}} Q\right\|_{\infty} \leq c_{Q}=2 \cdot 10^{-5} .
\end{aligned}
$$

and therefore,

$$
\left\|p_{2}(\widetilde{\mathcal{L}}-\mathcal{L}) \delta\right\|_{\infty} \leq c_{\widetilde{\mathcal{L}}}\|\delta\|, \quad c_{\widetilde{\mathcal{L}}}:=c_{P}+c_{Q}=4 \cdot 10^{-5} .
$$

\section{Linear stability of $f_{0}$}

In this section we prove that $f_{0}$ is linearly stable, apart from the gauge mode, in the sense that the self-adjoint operator $\mathcal{A}_{0}$, defined in (1.5), has no eigenvalues in the interval $(-\infty, 0]$ other then $\lambda_{0}=-1$. We divide the proof into two steps. First, we prove that $W_{0}$, defined as the solution of the initial value problem

$$
\mathcal{L} W_{\lambda}=\lambda W_{\lambda}, \quad W_{\lambda}(0)=0, \quad W_{\lambda}^{\prime}(0)=1
$$

with $\lambda=0$, has exactly one zero on $(0, \infty)$ (note that $W_{0}$ coincides with the fundamental solution $w_{0}$ up to normalization). Then, we prove that $W_{\lambda_{0}}(y):=y f_{0}^{\prime}(y)$ is an eigenfunction of $\mathcal{A}_{0}$ to the eigenvalue $\lambda_{0}=-1$. Finally, we apply the Sturm oscillation theorem, which relates the number of zeros of $W_{0}$ on $(0, \infty)$ to the number of eigenvalues below zero. Since 
$W_{0}$ has exactly one zero, there is only a single eigenvalue below zero. But this must be the eigenvalue $\lambda_{0}=-1$ to the gauge mode $W_{\lambda_{0}}$.

\subsection{Counting the zeros.}

Lemma 3.1. The solution $W_{0}$ of the initial value problem (3.1) with $\lambda=0$ has exactly one zero in $(0, \infty)$. Moreover,

$$
q(y):=W_{0}(y) / y
$$

is negative and decreasing for $y \geq 3$.

Proof. Let us note that the differential eqation for $W_{0}$ can be written in terms of $q$ as

$$
q^{\prime \prime}(y)=\frac{1}{2 y}\left(y^{2}-8\right)\left(q^{\prime}(y)+\frac{q(y)}{y}\right)+\frac{4}{y^{2}} \cos \left(\widetilde{f}_{0}(y)\right)^{2} q(y) .
$$

From the structure of the above equation one immediately notices that, if $q(y)<0$ and $q^{\prime}(y)<0$ for some $y \geq 3$, then $q^{\prime \prime}(y)<0$. Consequently, if $q(3)<0$ and $q^{\prime}(3)<0$, it follows that

$$
q(y)<0, \quad q^{\prime}(y)<0 \quad \text { for all } y \geq 3 .
$$

The second observation is that if $q(0)>0, q(3)<0$ and $q^{\prime}(y)<0$ for all $y \in[0,3]$ then the function $q$ has exactly one zero in $[0,3]$. In combination with (3.2) this implies that $q$ has exactly one zero in $[0, \infty)$ and thus, $W_{0}$ has exactly one zero in $(0, \infty)$. We shall now show that these preconditions do indeed occur for $q$.

First let us denote $\delta_{0}:=W_{0}-\widetilde{w}_{0}$, where $\widetilde{w}_{0}$ is the approximation to $w_{0}$ introduced in the previous section. Rewriting (3.1) for $\delta_{0}$ we have

$$
\mathcal{L} \delta_{0}=-\mathcal{L} \widetilde{w}_{0}, \quad \delta_{0}(0)=0, \quad \delta_{0}^{\prime}(0)=1-\widetilde{w}_{0}^{\prime}(0)
$$

or, equivalently,

$$
\widetilde{\mathcal{L}} \delta_{0}=-\mathcal{L} \widetilde{w}_{0}+(\widetilde{\mathcal{L}}-\mathcal{L}) \delta_{0}
$$

with the same initial condition. Note that one could easily normalize $\widetilde{w}_{0}^{\prime}(0)$ to one but this is not necessary for our line of reasoning. Eq. (3.3) can be treated with the machinery from the previous sections. However, before we proceed we need one more technical modification.

The main difference between (3.3) and (2.2) is that the source term $-\mathcal{L} \widetilde{w}_{0}$ in (3.3) is unbounded and grows exponentially as $y \rightarrow \infty$ and we are therefore unable to apply the

estimates on $\widetilde{\mathcal{L}}^{-1}$ from the previous section directly. To fix this issue we regularize the source term $-\mathcal{L} \widetilde{w}_{0}$ outside of $[0,3]$ by multiplying it with the indicator function

$$
\chi(y)= \begin{cases}1 & y \in[0,3] \\ 0 & y \in(3, \infty) .\end{cases}
$$

Let us now consider the solution $\widehat{\delta}_{0}$ to the ad-hoc regularized boundary value problem

$$
\begin{array}{r}
\widetilde{\mathcal{L}} \delta=-\chi \mathcal{L} \widetilde{w}_{0}+(\widetilde{\mathcal{L}}-\mathcal{L}) \delta, \\
\delta(0)=0, \quad \delta(\infty)=\text { const } .
\end{array}
$$

For the sake of the argument we will assume that the problem (3.4) can actually be solved (we prove this explicitly in the next paragraph). With the solution $\widehat{\delta}_{0}$ at hand, we define a 
function $\widehat{W}_{0}:=c_{0}\left(\widetilde{w}_{0}+\widehat{\delta}_{0}\right)$ with $c_{0}=1 /\left(\widetilde{w}_{0}^{\prime}(0)+\widehat{\delta}_{0}^{\prime}(0)\right)$, where we postpone the verification that $c_{0}$ is finite for the sake of clarity. By construction, $\widehat{W}_{0}$ solves the initial value problem

$$
\mathcal{L} \widehat{W}_{0}=c_{0}(1-\chi) \mathcal{L} \widetilde{w}_{0}, \quad \widehat{W}_{0}(0)=0, \quad \widehat{W}_{0}^{\prime}(0)=1 .
$$

Observe now that the right hand side of the differential equation in (3.5) is identically zero on $[0,3]$. This means that $W_{0}$ and $\widehat{W}_{0}$ coincide on $[0,3]$, i.e., $W_{0}=\widehat{W}_{0}=c_{0}\left(\widetilde{w}_{0}+\widehat{\delta}_{0}\right)$ on $[0,3]$. But the expression $c_{0}\left(\widetilde{w}_{0}+\widehat{\delta}_{0}\right)$ is actually semi-explicit: we know $\widetilde{w}_{0}$ explicitly and we will show that $\widehat{\delta}_{0}$ is small, which leads to precise pointwise bounds on $W_{0}$.

To show that there exists a solution $\widehat{\delta}_{0}$, we proceed as in the previous sections: we solve Eq. (3.4) with a fixed point argument applied to the map

$$
\mathcal{J}(\delta):=\widetilde{\mathcal{L}}^{-1}\left(-\chi \mathcal{L} \widetilde{w}_{0}+(\widetilde{\mathcal{L}}-\mathcal{L}) \delta\right)
$$

acting on the ball

$$
Y=\left\{\delta \in C^{1}([0, \infty)):\|\delta\| \leq 0.03\right\}
$$

Before we move on, let us take a closer look at the term $-\chi \mathcal{L} \widetilde{w}_{0}$. We constructed $\widetilde{w}_{0}$ so that $\widetilde{\mathcal{L}} \widetilde{w}_{0}=0$, thus

$$
-\chi \mathcal{L} \widetilde{w}_{0}=\chi(\widetilde{\mathcal{L}}-\mathcal{L}) \widetilde{w}_{0}=\chi\left(P \widetilde{w}_{0}^{\prime}+Q \widetilde{w}_{0}\right)=\left(P \chi \widetilde{w}_{0}^{\prime}+Q \chi \widetilde{w}_{0}\right) .
$$

Using this identity we infer

$$
\begin{aligned}
\left\|p_{2} \chi \mathcal{L} \widetilde{w}_{0}\right\|_{\infty} & =\left\|p_{2}\left(P \chi \widetilde{w}_{0}^{\prime}+Q \chi \widetilde{w}_{0}\right)\right\|_{\infty} \\
& \leq\left\|P \frac{p_{2}}{p_{3}}\right\|_{\infty}\left\|p_{3} \chi \widetilde{w}_{0}^{\prime}\right\|_{\infty}+\left\|Q \frac{p_{2}}{p_{1}}\right\|_{\infty}\left\|p_{1} \chi \widetilde{w}_{0}\right\|_{\infty} \\
& \leq c_{P}\left\|p_{3} \widetilde{w}_{0}^{\prime}\right\|_{L^{\infty}(0,3)}+c_{Q}\left\|p_{1} \widetilde{w}_{0}\right\|_{L^{\infty}(0,3)} \\
& =c_{\widetilde{\mathcal{L}}}\left(\frac{1}{2}\left\|p_{3} \widetilde{w}_{0}^{\prime}\right\|_{L^{\infty}(0,3)}+\frac{1}{2}\left\|p_{1} \widetilde{w}_{0}\right\|_{L^{\infty}(0,3)}\right) \\
& \leq c_{\widetilde{\mathcal{L}}} c_{\widetilde{w}_{0}},
\end{aligned}
$$

where $c_{\widetilde{w}_{0}}$ is a bound on the supremum, yet to be computed, and we made use of $c_{P}=c_{Q}=$ $c_{\widetilde{\mathcal{L}}} / 2$ (cf. section 2.4). From this bound we immediately infer

$$
\begin{aligned}
\|\mathcal{J}(\delta)\| & \leq c_{\mathcal{L}} c_{\widetilde{\mathcal{L}}}\left(c_{\widetilde{w}_{0}}+\|\delta\|\right), \\
\|\mathcal{J}(\delta)-\mathcal{J}(\gamma)\| & \leq c_{\mathcal{L}} c_{\widetilde{\mathcal{L}}}\|\delta-\gamma\|,
\end{aligned}
$$

for all $\delta, \gamma \in Y$, where $c_{\mathcal{L}} c_{\widetilde{\mathcal{L}}}=0.0072<1$. The only new estimate in (3.6) is the one for $c_{\widetilde{w}_{0}}$, but it can be readily computed using interval arithmetic. We have 3

$$
\left\|p_{3} \widetilde{w}_{0}^{\prime}\right\|_{L^{\infty}(0,3)} \leq 1.2, \quad\left\|p_{1} \widetilde{w}_{0}\right\|_{L^{\infty}(0,3)} \leq 4
$$

so that

$$
c_{\widetilde{w}_{0}}=\frac{1}{2}(1.2+4)=2.6
$$

\footnotetext{
${ }^{3}$ In the estimate (3.7) we are actually estimating a rational function times an exponential function. The interval arithmetic for rational functions, which we introduced earlier on via Definition 2.3 and Theorem 2.3 . can be easily extended to include operations on exponents by defining $\exp ([a, b]):=[\exp (a), \exp (b)]$ thanks to the monotonicity of exp.
} 
We conclude that the map $\mathcal{J}$ maps $Y$ into itself,

$$
\|\mathcal{J}(\delta)\| \leq c_{\mathcal{L}} c_{\widetilde{\mathcal{L}}}(2.6+0.03) \leq c_{\mathcal{L}} c_{\widetilde{\mathcal{L}}} \cdot 3=0.0216<0.03
$$

and thus, by the contraction mapping principle, we obtain the existence of $\widehat{\delta}_{0} \in Y$ solving (3.4). The normalization constant $c_{0}=1 /\left(\widetilde{w}_{0}^{\prime}(0)+\widehat{\delta}_{0}^{\prime}(0)\right)$, used in the definition of $\widehat{W}_{0}$, can now be easily verified to be finite and positive because, by explicit computation, $\widetilde{w}_{0}^{\prime}(0)>1$ and $\left|\widehat{\delta}_{0}^{\prime}(0)\right| \leq\left\|\widehat{\delta}_{0}\right\| \leq 0.03 \ll 1$.

As already mentioned, we have $W_{0}=\widehat{W}_{0}=c_{0}\left(\widetilde{w}_{0}+\widehat{\delta}_{0}\right)$ on $[0,3]$, so the estimate

$$
p_{1}\left|W_{0} / c_{0}-\widetilde{w}_{0}\right|+p_{3}\left|W_{0}^{\prime} / c_{0}-\widetilde{w}_{0}^{\prime}\right| \leq\left\|\widehat{\delta}_{0}\right\| \leq 0.03
$$

holds on $[0,3]$. In other words,

$$
\begin{gathered}
\widetilde{w}_{0}-0.03 / p_{1} \leq W_{0} / c_{0} \leq \widetilde{w}_{0}+0.03 / p_{1}, \\
\widetilde{w}_{0}^{\prime}-0.03 / p_{3} \leq W_{0}^{\prime} / c_{0} \leq \widetilde{w}_{0}^{\prime}+0.03 / p_{3}
\end{gathered}
$$

on $[0,3]$. From (3.8) we infer

$$
\begin{aligned}
q(3) & =W_{0}(3) / 3 \leq c_{0}\left(\widetilde{w}_{0}(3)+0.03 / p_{1}(3)\right) / 3 \leq-0.06 c_{0}<0, \\
q^{\prime}(3) & =W_{0}^{\prime}(3) / 3-W_{0}(3) / 3^{2} \\
& \leq c_{0}\left(\widetilde{w}_{0}^{\prime}(3)+0.03 / p_{3}(3)\right) / 3-c_{0}\left(\widetilde{w}_{0}(3)-0.03 / p_{1}(3)\right) / 3^{2} \leq-0.05 c_{0}<0 .
\end{aligned}
$$

Consequently, by our earlier result (3.2), $q$ has no zeros in $[3, \infty)$, nor has $W_{0}$. At the same time the interval arithmetic (with $W_{0}$ replaced by $\widetilde{w}_{0}$ according to the bounds (3.8)) reveals that

$$
\begin{aligned}
q(y)>0 & \text { for } y \in[0,1], \\
q^{\prime}(y)<0 & \text { for } y \in[1,3]
\end{aligned}
$$

which, in combination with $q(3)<0$, means that $q$ traverses zero exactly once on $(0, \infty)$, and so does $W_{0}$.

Remark 3.2. Since $\mathcal{L}$ is an approximation to $\mathcal{A}_{0}$, the same type of argument can be used to show that Lemma 3.1 holds for the solution $W_{0}$ of $\mathcal{A}_{0} W_{0}=0, W_{0}(0)=0, W_{0}^{\prime}(0)=1$.

\subsection{Applying the Sturm oscillation to $\mathcal{A}_{0}$.}

Lemma 3.3. The operator $\mathcal{A}_{0}$ has no eigenvalue at $\lambda=0$.

Proof. We will show that $W_{0} \notin \mathcal{H}$ and thus, $W_{0}$ is not an eigenfunction. The two possible behaviors of $W_{0}(y)$ when $y \rightarrow \infty$ are $W_{0}(y)=-1+\mathcal{O}\left(y^{-2}\right)$ or $W_{0}(y)=y^{-1} e^{y^{2} / 4}\left(1+\mathcal{O}\left(y^{-2}\right)\right)$ (both are up to normalization). Only the first, recessive, behavior leads to $W_{0} \in \mathcal{H}$. Assume that $W_{0}(y)=-1+\mathcal{O}\left(y^{-2}\right)$. Then $q(y)=-1 / y+\mathcal{O}\left(y^{-3}\right)$ and $q^{\prime}(y)=1 / y^{2}+\mathcal{O}\left(y^{-4}\right)$, so $q^{\prime}(y)>0$ for sufficiently large $y$. But this is a contradiction to $q(y)<0$ and $q^{\prime}(y)<0$ for $y \geq 3$ from Lemma 3.1. Thus, $W_{0}$ grows exponentially and $\lambda=0$ is not an eigenvalue.

Lemma 3.4. The function $W_{\lambda_{0}}(y):=y f_{0}^{\prime}(y)$ is an eigenfunction of $\mathcal{A}_{0}$ to the eigenvalue $\lambda_{0}=-1$. Furthermore, $W_{\lambda_{0}}>0$ on $(0, \infty)$. 
Proof. One can easily check that if $f_{0}$ solves (1.2) then

$$
W_{\lambda_{0}}(y):=y f_{0}^{\prime}(y)
$$

lies in $\mathcal{H}$ and solves the differential equation $\mathcal{A}_{0} W_{\lambda_{0}}=\lambda_{0} W_{\lambda_{0}}$. Therefore, $W_{\lambda_{0}}$ is an eigenfunction of $\mathcal{A}_{0}$ to the eigenvalue $\lambda_{0}=-1$. Moreover, from Theorem [1.1] we know that $f_{0}$ is close to its approximation $\widetilde{f}_{0}$ in the sense that

$$
\widetilde{f}_{0}^{\prime}(y)-5 \cdot 10^{-4} / p_{3}(y) \leq f_{0}^{\prime}(y) \leq \widetilde{f_{0}^{\prime}}(y)+5 \cdot 10^{-4} / p_{3}(y) .
$$

By applying this bound to the definition of $W_{\lambda_{0}}$ we get

$$
W_{\lambda_{0}}(y) \geq y \widetilde{f}_{0}^{\prime}(y)-5 \cdot 10^{-4} y / p_{3}(y) .
$$

The right hand side can be written explicitly in the form

$$
y \widetilde{f}_{0}^{\prime}(y)-5 \cdot 10^{-4} y / p_{3}(y)=x\left(1-x^{2}\right) \frac{\sum_{n=0}^{N} a_{n} x^{2 n}\left(1-x^{2}\right)^{N-n}}{\sum_{k=0}^{K} b_{k} x^{2 k}\left(1-x^{2}\right)^{K-k}}, \quad x=\frac{y}{\sqrt{2+y^{2}}}
$$

with $N, K=59$ and all $a_{n}, b_{k}>0$, ultimately yielding

$$
W_{\lambda_{0}}(y)>0, \quad y \in(0, \infty) .
$$

At this point, the proof of Theorem 1.6 reduces to an application of Sturm comparison and oscillation theorems. The comparison theorem is as follows.

Lemma 3.5. Let $W_{\lambda}$ be the unique solution of the initial value problem

$$
\mathcal{A}_{0} W_{\lambda}=\lambda W_{\lambda}, \quad W_{\lambda}(0)=0, \quad W_{\lambda}^{\prime}(0)=1 .
$$

If $\lambda \leq-1$ then $W_{\lambda}$ has no zeros in $(0, \infty)$.

Proof. We follow the proof in [25], Theorem 2.6.3. The case $\lambda=-1$ is handled by Lemma 3.4, so assume $\lambda<\lambda_{0}:=-1$. From Lemma 3.4 we know that $W_{\lambda_{0}}>0$ on $(0, \infty)$. Thus, $W_{\lambda} / W_{\lambda_{0}}$ is well-defined and a straightforward computation reveals the Picone identity

$$
\left[\rho \frac{W_{\lambda}}{W_{\lambda_{0}}}\left(W_{\lambda}^{\prime} W_{\lambda_{0}}-W_{\lambda} W_{\lambda_{0}}^{\prime}\right)\right]^{\prime}=\left(\lambda_{0}-\lambda\right) \rho W_{\lambda}^{2}+\rho \frac{\left(W_{\lambda}^{\prime} W_{\lambda_{0}}-W_{\lambda} W_{\lambda_{0}}^{\prime}\right)^{2}}{W_{\lambda_{0}}^{2}}
$$

with $\rho(y)=y^{2} e^{-y^{2} / 4}$. Now assume that the statement is wrong and $y_{0}>0$ is the first zero of $W_{\lambda}$ in $(0, \infty)$. Integrating the Picone identity from 0 to $y_{0}$ yields

$$
0=\left(\lambda_{0}-\lambda\right) \int_{0}^{y_{0}} W_{\lambda}(y)^{2} \rho(y) d y+\int_{0}^{y_{0}} \frac{\left[W_{\lambda}^{\prime}(y) W_{\lambda_{0}}(y)-W_{\lambda}(y) W_{\lambda_{0}}^{\prime}(y)\right]^{2}}{W_{\lambda_{0}}(y)^{2}} \rho(y) d y,
$$

where we have used $\rho(0)=W_{\lambda}\left(y_{0}\right)=0$. Consequently, since $\lambda_{0}-\lambda>0$, we infer $W_{\lambda}(y)=0$ for all $y \in\left[0, y_{0}\right]$, which implies that $W_{\lambda}$ is the trivial solution, a contradiction.

Now the proof of Theorem 1.6 is readily completed by invoking Theorem 1.2 from [21], which states that in the interval $[\lambda, 0), \lambda \leq-1$, there are exactly as many eigenvalues as there are zeros of $W_{0}$ minus the zeros of $W_{\lambda}$, that is, $1-0=1$. Consequently, there is exactly one eigenvalue in each interval $[\lambda, 0)$ for any $\lambda \leq-1$. This single eigenvalue has

\footnotetext{
${ }^{4}$ Note that there is a small typo in Theorem 1.2 in [21]: $N(c)$ is supposed to denote the number of zeros of $u_{2}$ minus the number of zeros of $u_{1}$, not the other way round.
} 
to be $\lambda_{0}=-1$ from Lemma 3.4. Furthermore, we have already proved that $W_{0}$ is not an eigenfunction, so the only eigenvalue in $(-\infty, 0]$ is $\lambda_{0}=-1$.

3.3. Proof of Proposition 1.3. The proof is an extended version of an argument given in [3]. We write the equation (1.2) as

$$
\frac{1}{\rho}\left(\rho f^{\prime}\right)^{\prime}=S_{1}, \quad S_{1}(y)=\frac{\sin (2 f(y))}{y^{2}}
$$

Differentiating (3.9) $k-1$ times we get

$$
\left(\frac{1}{\rho}\left(\rho f^{\prime}\right)^{\prime}\right)^{(k-1)}=S_{1}^{(k-1)}
$$

We then use the identity

$$
\left(\frac{1}{\rho}\left(\rho f^{\prime}\right)^{\prime}\right)^{\prime}(y)-\frac{1}{\rho}\left(\rho f^{\prime \prime}\right)^{\prime}(y)=-\left(\frac{2}{y^{2}}+\frac{1}{2}\right) f^{\prime}(y)
$$

to obtain a differential equation for $f^{\prime \prime}$, which reads

$$
\frac{1}{\rho}\left(\rho f^{\prime \prime}\right)^{\prime}(y)=S_{1}^{\prime}(y)+\left(\frac{2}{y^{2}}+\frac{1}{2}\right) f^{\prime}(y)=: S_{2}(y) .
$$

By repeating this procedure, we find a differential equation for the $k$-th derivative of $f$,

$$
\frac{1}{\rho}\left(\rho f^{(k)}\right)^{\prime}=S_{k}, \quad S_{k}(y)=S_{k-1}^{\prime}(y)+\left(\frac{2}{y^{2}}+\frac{1}{2}\right) f^{(k-1)}(y) .
$$

Multiplying (3.10) by $\rho$ and integrating on $[y, \infty)$ gives us

$$
\lim _{x \rightarrow \infty} \rho(x) f^{(k)}(x)-\rho(y) f^{(k)}(y)=\int_{y}^{\infty} \rho(x) S_{k}(x) d x
$$

It is easy to see that any derivative of $f$ will grow at most algebraically at infinity (this follows directly from (3.9) and its derivatives); at the same time $\rho(y)=y^{2} e^{-\frac{y^{2}}{4}}$ decays exponentially at infinity so the limit on the left hand side is zero and the integral on the right hand side of (3.10) converges. We thus end up with

$$
f^{(k)}(y)=\frac{-\int_{y}^{\infty} \rho(x) S_{k}(x) d x}{\rho(y)} .
$$

Assume now that $\left|f^{(n)}(y)\right| \lesssim y^{-2-n}$ for any $n=1, \ldots k-1$. Then it is easy to see that $\left|S_{k}(y)\right| \leq C_{k-1} y^{-1-k}$ for some constant $C_{k-1}>0$ because, in the leading order, $S_{k}$ consists of terms of the form $f^{(k-1)}(y)$ and $y^{-1-k} \sin (2 f(y))$. In turn, this implies

$$
\left|f^{(k)}(y)\right| \leq C_{k-1} \frac{\int_{y}^{\infty} \rho(x) x^{-1-k} d x}{\rho(y)} \leq C_{k-1} \frac{y^{-1-k} \int_{y}^{\infty} \rho(x) d x}{\rho(y)} \leq C_{k} y^{-2-k} .
$$

Consequently, the claim $\left|f^{(k)}(y)\right| \leq C_{k} y^{-2-k}$ for all $k \in \mathbb{N}$ follows inductively.

The statement $f^{(2 k)}(0)=0$ for all $k \in \mathbb{N}_{0}$ follows essentially from the reflection symmetry of Eq. (1.2) under $y \mapsto-y$ and is easily proved inductively; we omit the details. 


\section{Appendix A. Estimates on the RAtios of Fundamental SOlutions}

The proof that the ratios $\left\|\widetilde{w}_{1} / v_{1}\right\| \leq 1.01$ and $\left\|\widetilde{w}_{1}^{\prime} / v_{1}^{\prime}\right\| \leq 1.01$ is a straightforward application of interval arithmetic as both $w_{1}$ and $v_{1}$ are rational functions. Things get more complicated when we try to compare $\widetilde{w}_{0}$ and $v_{0}$ or $\widetilde{w}_{0}^{\prime}$ and $v_{0}^{\prime}$ because, by definition,

$$
v_{0}(y)=\frac{3}{y^{2}} e^{y^{2} / 4}\left(-y+\left(2+y^{2}\right) D_{+}(y / 2)\right)
$$

contains Dawson's integral. As far as we know there is no definition of interval arithmetic for Dawson's integral, so we will have to resort to a more analytical approach. The goal now is to find an approximation $\widetilde{v}_{0}$ to $v_{0}$ such that the ratio $\widetilde{w}_{0} / \widetilde{v}_{0}$ is rational, so that we can apply our interval arithmetic algorithm.

Let us remind that the Wronskian of $v_{0}$ and $v_{1}$ is

$$
v_{0} v_{1}^{\prime}-v_{0}^{\prime} v_{1}=W\left(v_{0}, v_{1}\right)=-6 y^{-2} e^{y^{2} / 4},
$$

or equivalently

$$
\left(\frac{v_{0}}{v_{1}}\right)^{\prime}-\frac{6 e^{y^{2} / 4}}{v_{1}(y)^{2} y^{2}}=0,
$$

so $v_{0}$ can be written in the form of the following integral

$$
v_{0}(y)=6 v_{1}(y) \int_{0}^{y} \frac{e^{x^{2} / 4}}{v_{1}(x)^{2} x^{2}} d x .
$$

Let us assume that we found a suitable approximation $\widetilde{v}_{0}$ such that

$$
\left(\frac{\widetilde{v}_{0}}{v_{1}}\right)^{\prime}-\frac{6 e^{y^{2} / 4}}{v_{1}(y)^{2} y^{2}}=-\frac{6 e^{y^{2} / 4} \varepsilon(y)}{v_{1}(y)^{2} y^{2}}
$$

with

$$
0 \leq \varepsilon(y) \leq c_{\varepsilon} \ll 1
$$

The integral representation of $\widetilde{v}_{0}$ is simply

$$
\begin{aligned}
\widetilde{v}_{0}(y) & =6 v_{1}(y) \int_{0}^{y} \frac{e^{x^{2} / 4}}{v_{1}(x)^{2} x^{2}}(1-\varepsilon(x)) d x \\
& =v_{0}(y)-6 v_{1}(y) \int_{0}^{y} \frac{e^{x^{2} / 4} \varepsilon(x)}{v_{1}(x)^{2} x^{2}} d x .
\end{aligned}
$$

which, after taking into account (A.4), leads to

$$
\left(1-c_{\varepsilon}\right) v_{0}(y) \leq \widetilde{v}_{0}(y) \leq v_{0}(y) .
$$

This means that finding $\widetilde{v}_{0}$ such that $\varepsilon$ is small in the sense of (A.4) can be directly translated to a pointwise bound on $v_{0}$. As for the estimate on the derivative $v_{0}^{\prime}$ we subtract (A.2) from (A.3) and get

$$
\widetilde{v}_{0}^{\prime}-v_{0}^{\prime}=-\frac{6 e^{y^{2} / 4}}{v_{1}(y) y^{2}} \varepsilon(y)+\frac{v_{1}^{\prime}(y)}{v_{1}(y)}\left(\widetilde{v}_{0}-v_{0}\right)
$$


The first term on the right hand side is negative and applying (A.5) to the second term (and remembering that $\left.v_{1}^{\prime}(y) / v_{1}(y)=-4 /\left(2 y+y^{3}\right)<0\right)$ we get

which implies

$$
\widetilde{v}_{0}^{\prime}-v_{0}^{\prime} \leq \frac{-c_{\varepsilon}}{1-c_{\varepsilon}} \frac{v_{1}^{\prime}}{v_{1}} \widetilde{v}_{0}
$$

$$
\frac{\widetilde{v}_{0}^{\prime}(y)}{v_{0}^{\prime}(y)} \leq \frac{1}{1+\frac{c_{\varepsilon}}{1-c_{\varepsilon}} \frac{v_{1}^{\prime}(y)}{v_{1}(y)} \frac{\widetilde{v}_{0}(y)}{\widetilde{v}_{0}^{\prime}(y)}}
$$

Note that $v_{0}^{\prime}, \tilde{v}_{0}^{\prime} \geq 0$.

To find the right approximation $\widetilde{v}_{0}$, we simply use a well established expression of $D_{+}$as a continued fraction ([16], formula (2.7)),

$$
D_{+}(z)=\frac{z}{1+2 z^{2}-} \frac{4 z^{2}}{3+2 z^{2}-} \frac{8 z^{2}}{5+2 z^{2}-} \frac{12 z^{2}}{7+2 z^{2}-} \ldots
$$

and plug it directly into the definition of $v_{0}$. Truncating (A.7) at the twelfth term we find, via interval arithmetic, that the associated $\widetilde{v}_{0}$ has

$$
0 \leq \varepsilon(y) \leq c_{\varepsilon}=\frac{1}{500}
$$

so from (A.5) we have

$$
\frac{\widetilde{w}_{0}(y)}{v_{0}(y)} \leq \frac{\widetilde{w}_{0}(y)}{\widetilde{v}_{0}(y)}
$$

As for the ratio of derivatives we use (A.6) to get

$$
\frac{\widetilde{w}_{0}^{\prime}(y)}{v_{0}^{\prime}(y)} \leq \frac{\widetilde{w}_{0}^{\prime}(y)}{\widetilde{v}_{0}^{\prime}(y)} \cdot \frac{1}{1+\frac{c_{\varepsilon}}{1-c_{\varepsilon}} \frac{v_{1}^{\prime}(y)}{v_{1}(y)} \frac{\widetilde{v}_{0}(y)}{\widetilde{v}_{0}^{\prime}(y)}} .
$$

The functions on the right hand sides of (A.8) and (A.9) are now explicit rational functions and they can be easily estimated by interval arithmetic which yields

$$
\frac{\widetilde{w}_{0}(y)}{v_{0}(y)} \leq 1.01, \quad \frac{\widetilde{w}_{0}^{\prime}(y)}{v_{0}^{\prime}(y)} \leq 1.01 .
$$

Since all functions with subscript 0 are nonnegative, the claimed bounds follow. 


\section{ApPendix B. TABles of COEFFicients FOR APPROXimate SOLUtions}

TABLE B.1. The coefficients of the approximate solution $\widetilde{f}_{0}$

\begin{tabular}{c|cr|c}
$n$ & $\left(f_{0}\right)_{n}$ & $n$ & $\left(f_{0}\right)_{n}$ \\
\hline 0 & $\frac{268245}{72878}$ & 8 & $\frac{1}{204079}$ \\
1 & $\frac{-3774}{105551}$ & 9 & $\frac{1}{675805}$ \\
2 & $\frac{1897}{97022}$ & 10 & $\frac{1}{140761}$ \\
3 & $\frac{14}{72731}$ & 11 & $\frac{1}{3586839}$ \\
4 & $\frac{79}{119383}$ & 12 & $\frac{1}{7289041}$ \\
5 & $\frac{4}{66337}$ & 13 & $\frac{1}{16940631}$ \\
6 & $\frac{5}{109368}$ & 14 & $\frac{1}{59286294}$ \\
7 & $\frac{1}{109045}$ & &
\end{tabular}

TABLE B.2. The coefficients of $\widetilde{w}_{0}$

\begin{tabular}{|c|c|c|c|c|c|c|c|}
\hline$n$ & $\left(w_{0}\right)_{n}$ & $n$ & $\left(w_{0}\right)_{n}$ & $n$ & $\left(w_{0}\right)_{n}$ & $n$ & $\left(w_{0}\right)_{n}$ \\
\hline 0 & $\frac{18741}{112373}$ & 1 & $\frac{-61989}{17060}$ & 2 & $\frac{2353}{10197}$ & 3 & $\frac{-9791}{92415}$ \\
\hline 4 & $\begin{array}{l}\frac{27966}{4407} \\
\frac{1}{4407}\end{array}$ & 5 & $\begin{array}{l}-1970 \\
541159\end{array}$ & 6 & $\begin{array}{l}\frac{723}{52439} \\
5 \frac{10}{5}\end{array}$ & 7 & 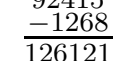 \\
\hline 8 & $\frac{1160}{216201}$ & 9 & $\frac{-52}{32323}$ & 10 & $\frac{113}{60053}$ & 11 & $\frac{-66}{11007}$ \\
\hline 12 & $\frac{21}{9801}$ & 13 & $\frac{-19}{49629}$ & 14 & $\frac{-2}{77991}$ & 15 & $\frac{-4}{43893}$ \\
\hline 16 & $\frac{2}{43899}$ & 17 & $\frac{1}{42980}$ & 18 & $\frac{4}{101127}$ & 19 & $\frac{1}{77370}$ \\
\hline 20 & $\frac{1}{10203}$ & 21 & $\frac{-1}{160116}$ & 22 & $\frac{-1}{105707}$ & 23 & $\frac{-1}{165215}$ \\
\hline 24 & $\frac{-1}{326029}$ & 25 & $\frac{-1}{101520}$ & 26 & $\frac{1}{105701}$ & 27 & $\frac{1}{8000}$ \\
\hline 28 & $\frac{1}{855294}$ & 29 & $\frac{1}{135569}$ & 30 & $\frac{1}{201711}$ & 31 & $\frac{-1}{\frac{802012}{10070}}$ \\
\hline 32 & $\frac{-1}{5479794}$ & 33 & $\frac{-1}{447515}$ & 34 & $\frac{-1}{567792}$ & 35 & -1 \\
\hline 36 & $\frac{-1}{-1}$ & 37 & $\begin{array}{r}\quad 1150 \\
\\
\end{array}$ & 38 & 1 & 39 & $\begin{array}{l}900 / 118 \\
\end{array}$ \\
\hline 40 & $\begin{array}{l}3283728 \\
\end{array}$ & 41 & $\begin{array}{l}5453641 \\
\\
\end{array}$ & 42 & $\begin{array}{c}2202853 \\
\end{array}$ & 43 & $\begin{array}{r}2032064 \\
1\end{array}$ \\
\hline 44 & $\begin{array}{l}1 \\
1 \\
1.1800\end{array}$ & & & & & & \\
\hline
\end{tabular}


TABLE B.3. The coefficients of $\widetilde{w}_{1}$

\begin{tabular}{r|cr|cr|cr|c}
$n$ & $\left(w_{1}\right)_{n}$ & $n$ & $\left(w_{1}\right)_{n}$ & $n$ & $\left(w_{1}\right)_{n}$ & $n$ & $\left(w_{1}\right)_{n}$ \\
\hline 0 & $\frac{4607589}{9727120}$ & 1 & $\frac{1737631}{2734940}$ & 2 & $\frac{-3983}{1039272}$ & 3 & $\frac{-256739}{1728298}$ \\
4 & $\frac{18231}{688103}$ & 5 & $\frac{212793}{6236542}$ & 6 & $\frac{-66549}{3547063}$ & 7 & $\frac{-1981}{1692925}$ \\
8 & $\frac{13293}{2855801}$ & 9 & $\frac{-10983}{6516796}$ & 10 & $\frac{-28}{197979}$ & 11 & $\frac{1701}{5019274}$ \\
12 & $\frac{-525}{3869749}$ & 13 & $\frac{147}{5955391}$ & 14 & $\frac{3}{664118}$ & 15 & $\frac{-63}{6270472}$ \\
16 & $\frac{21}{2638831}$ & 17 & $\frac{-7}{2307718}$ & 18 & $\frac{-7}{18723692}$ & 19 & $\frac{7}{6169514}$ \\
20 & $\frac{-21}{34570120}$ & 21 & $\frac{7}{84719934}$ & 22 & $\frac{3}{31279664}$ & 23 & $\frac{-21}{272250212}$ \\
24 & $\frac{3}{117220480}$ & 25 & $\frac{21}{13759709834}$ & 26 & $\frac{-21}{2997459842}$ & 27 & $\frac{7}{1630774626}$ \\
28 & $\frac{-7}{6156689032}$ & 29 & $\frac{-21}{64552514338}$ & 30 & $\frac{21}{41777614736}$ & 31 & $\frac{-21}{84914413922}$ \\
32 & $\frac{21}{547142712584}$ & 33 & $\frac{3}{84895626842}$ & 34 & $\frac{-3}{87634052792}$ & 35 & $\frac{1}{80692012804}$
\end{tabular}




\section{REFERENCES}

[1] Sigurd B. Angenent, Josephus Hulshof, and Hiroshi Matano. The radius of vanishing bubbles in equivariant harmonic map flow from $D^{2}$ to $S^{2}$. SIAM Journal on Mathematical Analysis, 41(3):1121-1137, 2009.

[2] Paweł Biernat. Non-self-similar blow-up in the heat flow for harmonic maps in higher dimensions. Nonlinearity, 28(1):1-26, 2015.

[3] Paweł Biernat and Piotr Bizoń. Shrinkers, expanders, and the unique continuation beyond generic blowup in the heat flow for harmonic maps between spheres. Nonlinearity, 24(8):2211-2228, 2011.

[4] Paweł Biernat, Roland Donninger, and Birgit Schörkhuber. Stable self-similar blowup in the supercritical heat flow of harmonic maps. Calc. Var. Partial Differential Equations, 56(6):Art. 171, 31, 2017.

[5] Paweł Biernat and Yukihiro Seki. Type II blow-up mechanism for supercritical harmonic map heat flow. Preprint arXiv:1601.01831, 2016.

[6] Piotr Bizoń and Arthur Wasserman. Nonexistence of Shrinkers for the Harmonic Map Flow in Higher Dimensions. International Mathematics Research Notices, 2015(17):7757-7762, 2015.

[7] Yunmei Chen and Michael Struwe. Existence and partial regularity results for the heat flow for harmonic maps. Mathematische Zeitschrift, 201(1):83-103, 1989.

[8] Ovidiu Costin, Min Huang, and Wilhelm Schlag. On the spectral properties of $L_{ \pm}$in three dimensions. Nonlinearity, 25(1):125-164, 2012.

[9] Matthew Creek, Roland Donninger, Wilhelm Schlag, and Stanley Snelson. Linear stability of the skyrmion. Int. Math. Res. Not. IMRN, (8):2497-2537, 2017.

[10] Roland Donninger and Birgit Schörkhuber. Stable blowup for the supercritical Yang-Mills heat flow. Preprint arXiv:1604.07737, 2016.

[11] James Eells and J. H. Sampson. Harmonic Mappings of Riemannian Manifolds. American Journal of Mathematics, 86(1):109, 1964.

[12] Huijun Fan. Existence of the self-similar solutions in the heat flow of harmonic maps. Science in China Series A: Mathematics, 42(2):113-132, 1999.

[13] Andreas Gastel. Singularities of first kind in the harmonic map and Yang-Mills heat flows. Mathematische Zeitschrift, 242(1):47-62, 2002.

[14] Pierre Germain, Tej-Eddine Ghoul, and Hideyuki Miura. On uniqueness for the harmonic map heat flow in supercritical dimensions. Preprint arXiv:1601.06601, 2016.

[15] Pierre Germain and Melanie Rupflin. Selfsimilar expanders of the harmonic map flow. Annales de l'Institut Henri Poincare (C) Non Linear Analysis, 28(5):743-773, 2011.

[16] J. H. McCabe. A continued fraction expansion, with a truncation error estimate, for Dawson's integral. Mathematics of Computation, 28(127):811-816, 1974.

[17] Pavol Quittner. Uniqueness of singular self-similar solutions of a semilinear parabolic equation. Preprint arXiv:1605.07394, 2016.

[18] Pierre Raphaël and Remi Schweyer. Stable blowup dynamics for the 1-corotational energy critical harmonic heat flow. Communications on Pure and Applied Mathematics, 66(3):414-480, 2013.

[19] Pierre Raphaël and Remi Schweyer. Quantized slow blow-up dynamics for the corotational energycritical harmonic heat flow. Analysis \& PDE, 7(8):1713-1805, 2014.

[20] Michael Reed and Barry Simon. Methods of modern mathematical physics II. Academic Press, 1975.

[21] Barry Simon, Gerald Teschl, and Fritz Gesztesy. Zeros of the Wronskian and renormalized oscillation theory. American Journal of Mathematics, 118(3):571-594, 1996.

[22] Michael Struwe. The evolution of harmonic maps: Existence, partial regularity, and singularities. In Nonlinear Diffusion Equations and Their Equilibrium States, 3, pages 485-491. 1989.

[23] Warwick Tucker. Validated Numerics for Pedestrians. In European Congress of Mathematics Stockholm, June 27 - July 2, 2004, pages 851-860. European Mathematical Society Publishing House, Zuerich, Switzerland.

[24] Warwick Tucker. Validated Numerics: A Short Introduction to Rigorous Computations. Princeton University Press, 2011.

[25] Anton Zettl. Sturm-Liouville theory, volume 121 of Mathematical Surveys and Monographs. American Mathematical Society, Providence, RI, 2005. 
Rheinische Friedrich-Wilhelms-Universität Bonn, Mathematisches Institut, Endenicher Allee 60, D-53115 Bonn, Germany

E-mail address: biernat@math.uni-bonn.de

Rheinische Friedrich-Wilhelms-Universität Bonn, Mathematisches Institut, Endenicher Allee 60, D-53115 Bonn, Germany

Universität Wien, Fakultät für Mathematik, Oskar-Morgenstern-Platz 1, A-1090 Vienna, Austria

E-mail address: donninge@math.uni-bonn.de 